\title{
Large area patterning of ultra-high thermal-stable structural colors in transparent solids
}

\author{
Jie Zhang (张 捷) ${ }^{1}$, Dezhi Tan (谭德志) $)^{2^{*}}$, Kaiqiang Cao (曹凯强) ${ }^{3}$, Tianqing Jia (贾天卿) ${ }^{3}$, and Jianrong Qiu (邱建荣) \\ ${ }^{1}$ State Key Laboratory of Modern Optical Instrumentation, College of Optical Science and Engineering, Zhejiang University, Hangzhou 310027, China \\ ${ }^{2}$ Zhejiang Lab, Hangzhou 311100, China \\ 3 State Key Laboratory of Precision Spectroscopy, School of Physics and Electronic Science, East China Normal University, Shanghai 200241 , China \\ ${ }^{4}$ CAS Center for Excellence in Ultra-Intense Laser Science, Chinese Academy of Sciences, Shanghai 201800, China
}

*Corresponding author: wctdz@zju.edu.cn

**Corresponding author: qji@zju.edu.cn

Received November 4, २०२1 | Accepted December 29, २०२१ | Posted Online January १२, २०२२

\begin{abstract}
Printing stable color with a lithography-free and environment-friendly technique is in high demand for applications. We report a facile strategy of ultrafast laser direct writing (ULDW) to produce large-scale embedded structural colors inside transparent solids. The diffraction effect of gratings enables effective generation of structural colors across the entire visible spectrum. The structural colors inside the fused silica glass have been demonstrated to exhibit excellent thermal stability under high temperature up to $1200^{\circ} \mathrm{C}$, which promises that the written information can be stable for long time even with unlimited lifetime at room temperature. The structural colors in the applications of coloring, anti-counterfeiting, and information storage are also demonstrated. Our studies indicate that the presented ULDW allows for fabricating large-scale and high thermal-stability structural colors with prospects of three-dimensional patterning, which will find various applications, especially under harsh conditions such as high temperature.
\end{abstract}

Keywords: ultrafast laser direct writing; structural color; glass; information storage.

DOI: 10.3788/COL202220.030501

\section{Introduction}

Structural colors have attracted conspicuous interest due to their fascinating potential in the applications of sensors, optical data storage, information encryption, and display devices. Now, vivid structural colors can be generated through the interaction of light with periodic structures-most of their period $(d)$ is comparable to the light wavelength ${ }^{[1-3]}$. Although as frequently suggested that the structural colored materials show superior stability compared with the colored chemical dyes and pigments, their stability at high temperature (e.g., $>700^{\circ} \mathrm{C}$ ) is intrinsically poor due to the large surface area of the sub-micrometer structures. Furthermore, nearly all of the current structural colors materials are built on substrates with the surface exposed to the environment, and this is inevitably detrimental to the structure stability. Stable structural colors at high temperature will be critical for storing the information for a long time ${ }^{[4]}$. Various techniques have been developed to fabricate desirable periodic structures for modulating the light field and producing structural colors, such as self-assembly and nanolithography ${ }^{[5-14]}$. However, complicated procedures are generally needed in most of the traditional fabrication methods, and limited scalability and lack of robustness still remain major challenges for the real-world applications.

Recently, ultrafast laser direct writing (ULDW) has been established to be a versatile strategy to induce periodic structures on the surface of many materials, such as metals, semiconductors, and dielectric solids, which usually rely on interference between multiple incident light beams or incident light and scattering light ${ }^{[5,15-18]}$. Typically, the laser induced periodic surface structures (LIPSSs) can exhibit obvious structural colors. Similarly, large-scale patterning periodic structures for structural colors with high thermal stability is also tough work.

Here, we demonstrate a simple strategy of ULDW to produce embedded microgratings with $d$ of several micrometers inside various glasses, which display bright structural colors under white light irradiation. The structural colors are revealed to originate from the diffraction effect. More importantly, the as-prepared structural colors in the fused silica glass can be stable up to $1200^{\circ} \mathrm{C}$, which has never been reported, and promises that the structural colors can be stable for a long time even with unlimited lifetime at room temperature. We have written colorful patterns with tunable periodic structures that indicate that the current structural colors have great potential in the 
applications of consumer product decoration, encryption, and optical data storage.

Diffraction of microgratings is a well-known principle that enables generation of structural colors. In contrast to the traditional cases to fabricate gratings with the feature size in the submicrometer range, we propose a simple technique of ULDW to write microgratings embedded in the transparent matrix and allow for efficiently producing structural colors with angle dependence. In this case, no any difficult-handed technique and complicated principle are needed, which can reduce the cost and improve the re-productivity for real-world applications.

\section{Materials and Methods}

In this study, microgratings are written in commercially available aluminosilicate (AS) glass (bandgap: $4 \mathrm{eV}$, melting temperature: $945.8^{\circ} \mathrm{C}$, composition: $90 \mathrm{SiO}_{2}-15 \mathrm{Al}_{2} \mathrm{O}_{3}-4 \mathrm{MgO}-$ $2 \mathrm{~K}_{2} \mathrm{O}-10 \mathrm{Na}_{2} \mathrm{O}$ ) and fused silica glass by a ultrafast laser. In experiments, an ultrafast laser with a repetition rate of $100 \mathrm{kHz}$, pulse duration of $1 \mathrm{ps}$, and wavelength of $1030 \mathrm{~nm}$ has been used. A microscope objective with $50 \times$ magnification (NA 0.8) is used to focus the laser beam into the glass beneath the surface of $150 \mu \mathrm{m}$, and the scanning speed is $1 \mathrm{~mm} / \mathrm{s}$. The optical micrographs are photograghed by a high-resolution Olympus SC180 color microscope camera, and the colorful images are obtained by an iPhone 12 camera. A wide spectrum light source in the range of $400-2200 \mathrm{~nm}$ is applied to irradiate the samples vertically, and the diffraction spectra are measured by a fiber spectrometer at different inspecting angles. Raman spectra are recorded with the exciting laser of $532 \mathrm{~nm}$.

\section{Results and Discussions}

We write a series of microgratings in the area of $2 \mathrm{~mm} \times 2 \mathrm{~mm}$ with diverse periods, which are 4.0, 3.6, 3.4, 3.2, 3.0, and $2.8 \mu \mathrm{m}$, in the same piece of AS glass by an ultrafast laser with pulse energy of $3 \mu \mathrm{J}$. The structural colors with angle dependence shown in Fig. 1(a) can be observed with the transmission of white light. Furthermore, from the left to right side, there is a significant blue shift with a decrease in the period $(d)$ from $4.0 \mu \mathrm{m}$ to $2.8 \mu \mathrm{m}$ and the same inspecting angle $(\theta)$. From bottom to top, a prominent red shift of the color is identified in the area with an increase in the inspecting angle $(\theta)$ and the same periodicity. Figure 1(b) shows the optical image of the micrograting with the period of $3.0 \mu \mathrm{m}$. We suggest that the structural colors originate from the diffraction effect of microgratings. When white light vertically transmits through the gratings with $d$ of $3.0 \mu \mathrm{m}$, the color spectral band is observed, as shown in Fig. 1(c), which is expected from the micrograting diffraction effect. The minimum period of the grating we made is $500 \mathrm{~nm}$, which exhibits unclear structural colors. This can be attributed to the small refractive index difference that is not enough to generate obvious grating effects.

For gratings illuminated by a white light beam vertically, the central wavelength of the diffracted light at a certain angle follows a simple formula:



Fig. 1. (a) Structural colors of microgratings written in AS glass with diverse periods (4.0-2.8 $\mu \mathrm{m}$ ) under white light irradiation. (b) Optical micrograph of a grating with $d$ of $3.0 \mu \mathrm{m}$. (c) The diffraction band behind the glass of (b) with white light irradiation.

$$
d \sin \theta=m \lambda,
$$

where $\lambda$ is the central wavelength, $d$ is the grating period, $\theta$ is the inspecting angle, and $m$ is the diffraction order.

Figure 2(a) shows the diffraction spectra of the second order ( $m=2$ and $\theta=20^{\circ}$ ) with an altering $d$. As $d$ increases from 2.5 to $4.0 \mu \mathrm{m}$, the corresponding $\lambda$ red shifts from 410 to $684 \mathrm{~nm}$. Theoretically, we can get $\lambda=(\sin \theta / m) \cdot d=k_{1} \cdot d$ from the diffraction equation, where $k_{1}=\sin \theta / m$, and it is in line with Fig. 2(b). Fixing the diffraction order and detecting angle, $\lambda$ of the band linearly increases with $d$, and the $k_{1}$ is determined to be 1.71 . To directly illustrate the rich colors achieved from our structures, each spectrum is converted into the RGB color value $^{[1-3,19-23]}$. The Commission on Illumination (CIE) 1931 chromaticity coordinates corresponding to the generated colors with diverse grating periods are illustrated in Fig. 2(c). The saturation of the structural colors is relatively high, and it covers a wide gamut.

Figure 2(d) shows the diffraction spectra of the first order ( $m=1, d=2 \mu \mathrm{m}$ ) at altering $\theta$. The corresponding $\lambda$ increases from 430 to $684 \mathrm{~nm}$ with $\theta$ increasing from $12^{\circ}$ to $20^{\circ}$. Figure 2(e) demonstrates that there is a linear increase in the central wavelength with an increase in the detecting angle, and it is consistent with the theoretical expectation. Here, $\lambda=(d / m) \cdot \sin \theta=k_{2} \cdot \sin \theta$, where $k_{2}=d / m=2$. The CIE chromaticity coordinates of the colors displayed in the spectrogram with altering $\theta$ are shown in Fig. 2(f).

To explore the thermal stability of the micrograting inside AS glass, gratings with $d$ of $4 \mu \mathrm{m}$ are treated at high temperature. Figures 3(a) and 3(b) are the optical images of the microgratings before and after heat treatment, respectively. The micrograting 


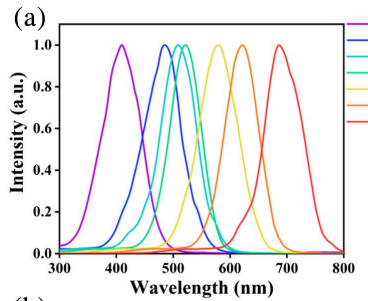

(b)
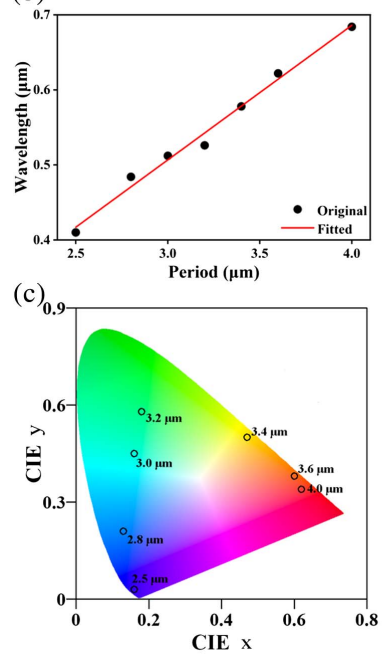

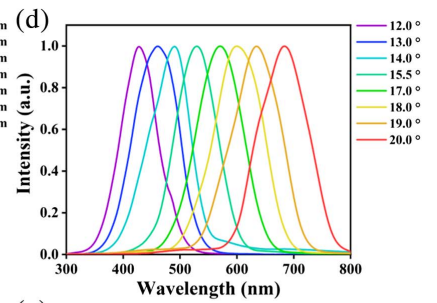

(e)

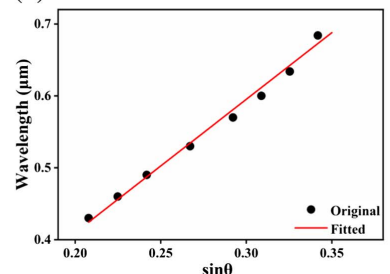

(f)

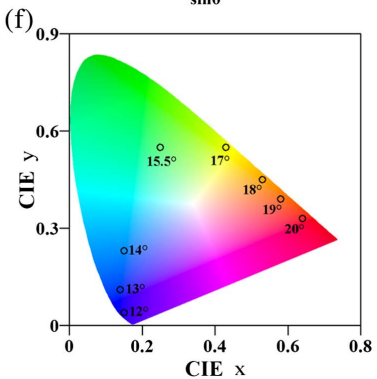

Fig. 2. (a) Diffraction spectra of samples with different $d$ irradiated by white light vertically measured at $\theta=20^{\circ}$. (b) Fitting curve of $d$ and the corresponding $\lambda$ in (a). (c) CIE1931 chromaticity diagram for the spectra in (a). (d) Diffraction spectra of a sample with $d=2.0 \mu \mathrm{m}$ irradiated by white light vertically measured at altering $\theta$. (e) Fitting curve of $\sin \theta$ and $\lambda$ in (d). (f) CIE1931 plot for the spectra in (d).

structure still exists after heat treatment at $750^{\circ} \mathrm{C}$ for $2 \mathrm{~h}$. Furthermore, the structural color is observable after heat treatment revealed in the inset of Fig. 3(b). Therefore, our studies indicate that the microgratings written inside AS glass are very stable at high temperature up to $750^{\circ} \mathrm{C}$.

We have also verified the current technique of ULDW is generally used to produce diffraction microgratings for structural colors in other glasses, such as fused silica glass and glass slide (composition: $100 \mathrm{SiO}_{2}-\mathrm{Al}_{2} \mathrm{O}_{3}-4 \mathrm{MgO}-14 \mathrm{CaO}-17 \mathrm{Na}_{2} \mathrm{O}$ ). We found that the repetition rates and pulse widths only affected the width and depth of grating lines, but did not affect the generating of the structural colors. It is reasonable to propose that as long as the laser parameters are optimized, we can write structural colors in nearly all of the transparent matrix. Moreover, the structural colors in fused silica glass can be stable at up to $1200^{\circ} \mathrm{C}$. We also confirmed that the structural colors exhibit the same stability at high temperature for the microgratings with different periodicities. Considering the chemical stability of glass, the embedded structural colored microgratings promise various applications under harsh conditions.

Raman spectra of the microgratings written with the pulse energy of 3,4 , and $5 \mu \mathrm{J}$ and written by $4 \mu \mathrm{J}$ pulse energy with heat treatment at $750^{\circ} \mathrm{C}$ for $2 \mathrm{~h}$ are measured to determine the formation mechanism of the gratings in glass, as shown in Fig. 3(c). Compared with the glass matrix, a new Raman peak

(a)

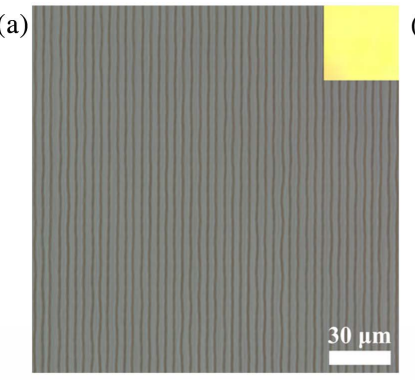

(b)

(c)

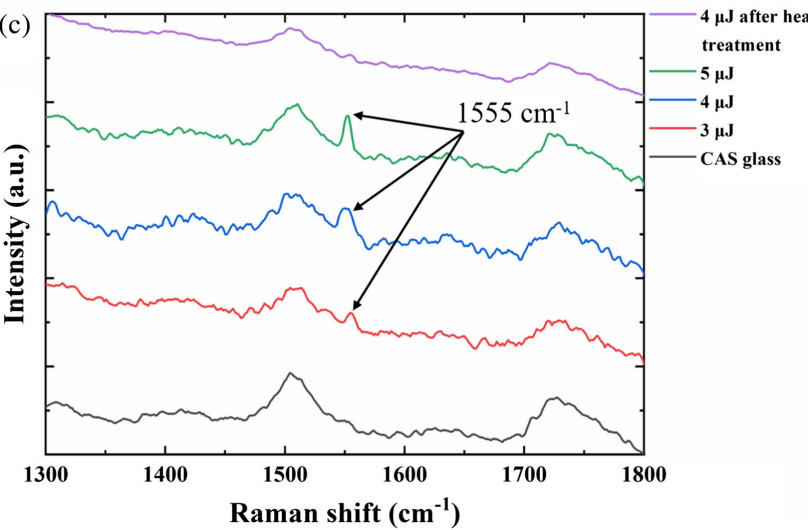

Fig. 3. Optical micrographs of $4 \mu \mathrm{m}$ microgratings with $4 \mu$ laser energy inside AS glass (a) before and (b) after heat treatment at $750^{\circ} \mathrm{C}$ for $2 \mathrm{~h}$. The insets at the top right in (a) and (b) represent the structural color observed at $\theta=20^{\circ}$ under white light, which are both $1 \mathrm{~mm} \times 1 \mathrm{~mm}$. (c) Raman spectra of (b), with AS glass and the microgratings written by diverse laser energy.

at $1555 \mathrm{~cm}^{-1}$ for the gratings appears, assigned to the natural vibration of $\mathrm{O}_{2}$ molecules, and the intensity of the peak increases with the increase of pulse energy ${ }^{[24-26]}$. Plasma with high temperature and high pressure in the local area of the glass can be induced by an ultrafast laser, in which there are plenty of highly ionized species, including $\mathrm{O}$ ions. As a result, molecular oxygen may form during the writing process. The Raman peak at $1555 \mathrm{~cm}^{-1}$ disappears after heat treatment, but the micrograting structure and the structural colors are still observable. Consequently, the formation of the micrograting structure is suggested to originate from ultrafast laser induced decomposition of the glass structure and the resultant change in refractive index ${ }^{[27-29]}$.

We demonstrate the realization of printing structural colored patterns composed of microgratings with different periods. Figures 4 (a) and 4 (b) are the design schematic diagrams of the Huawei icon and BIT logo. Roman numerals of I, II, III, IV, V, VI, and VII represent the gratings with a period of 4.0, $3.6,3.4,3.2,3.0,2.8$, and $2.5 \mu \mathrm{m}$, respectively. According to the design diagrams, we print the Huawei icon and BIT logo inside AS glass [Fig. 4(c)] and fused silica glass [Fig. 4(d)], respectively. The colorful Huawei icon and BIT logo observed depend on the diffraction angle.

The direction of the microgratings printed above is all parallel. We write two groups of microgratings perpendicular to each other and overlapped in an area. In this way, we can print two 
(a)
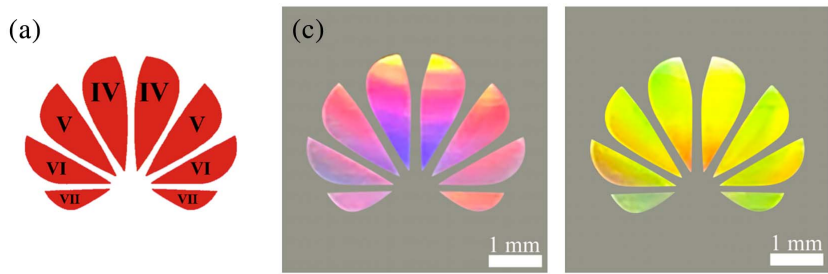

(b)

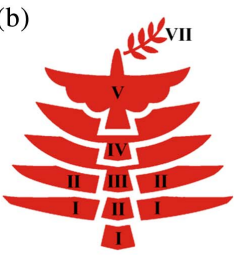

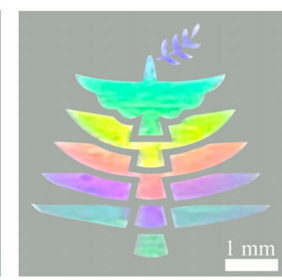

Fig. 4. Apply this technique to printing colorizing patterns. Schematic diagrams of (a) Huawei icon and (b) BIT logo. (c) Huawei icon and (d) BIT logo inside AS glass photographed under white light from different incident angles.

types of quick response $(\mathrm{QR})$ codes in the same region, as illustrated in Fig. 5(a). Figure 5(b) shows that two colorizing patterns of $\mathrm{QR}$ codes in the same area of $4.2 \mathrm{~mm} \times 4.2 \mathrm{~mm}$ are selectively displayed when this sample is irradiated by white light from different directions. Besides, microgratings with arbitrary directions can also be displayed with illumination in directions perpendicular to the corresponding grating lines ${ }^{[8,30]}$. However, if the angle of two gratings is not $90^{\circ}$, the reading light of one grating pattern will read out another grating pattern to a certain extent due to the vectorial property of light. Therefore, the ideal case is that the angle of two gratings equals $90^{\circ}$.

Our work indicates that ULDW structural colors hold great potential in the applications of coloring, anti-counterfeiting, and data storage. For example, logos and copyright patterns can be printed inside the consumer products for decoration and anti-counterfeiting. It is also possible to be applied to flexible structural color display, where the colors can be modified by altering the angle of the incident light. Furthermore, the high thermal stability of the structural colors promises that the written information can be stable for a long time even with unlimited lifetime at room temperature. It is worth noting that the AS glass adopted here is a typical glass of commercially available glass for

(a)

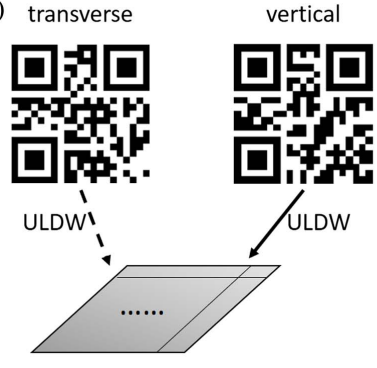

(b)

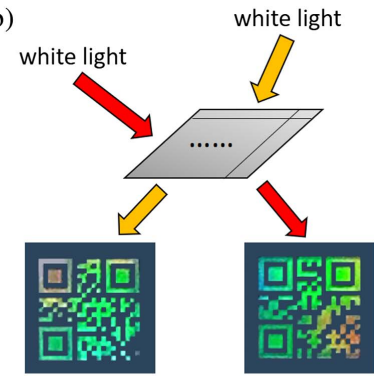

Fig. 5. (a) Two QR codes with different information, which are "ZJU" and "BIT" written in perpendicular directions. (b) Colorizing patterns of QR codes in (a) are selectively displayed in the same region with incident light from diverse angles. The patterns are of the dimension $4.2 \mathrm{~mm} \times 4.2 \mathrm{~mm}$.

the mobile phone cover plate. Consequently, it is valuable to print desirable patterns for personal customization needs in the glass cover plate. The feature of direct writing also holds the prospect of three-dimensional patterning. In addition, the writing scanning speed for the current work is limited by the translation stage, which is driven by piezoelectric ceramics and produced by SmarAct (Germany), designed for nano precise positioning. It is expected to more easily achieve large-scale patterning with a high scanning speed stage or a parallel writing system, which will enhance the efficiency and reduce the cost.

\section{Conclusions}

We have verified a facile technique of ULDW to fabricate large area microgratings embedded in various transparent matrices, which show rich structural colors with angle dependence. Moreover, our proposed structural colors exhibit excellent thermal stability, which enables the written information to be kept for a long time at room temperature. The combination of thermal stability and color saturation in the transparent matrix makes these structural colors suitable for the applications of consumer product decoration, which are proved by the display of various patterns with the structural colors. We also revealed two QR code patterns composed of spatially overlapped gratings with different directions in the same area, which can display, respectively, by changing the incident direction of white light. Therefore, this technique has great potential in the applications of anti-counterfeiting, information storage, and more fields besides decoration.

\section{Acknowledgement}

This work was financially supported by the National Key R\&D Program of China (No. 2021YFB2800500), National Natural Science Foundation of China (Nos. U20A20211, 51902286, 61775192, 61905215, and 51772270), Open Funds of the State Key Laboratory of High Field Laser Physics, Shanghai Institute of Optics and Fine Mechanics, Chinese Academy of Sciences, and Fundamental Research Funds for the Central Universities.

\section{References}

1. H. Pan, Z. Wen, Z. Tang, G. Xu, X. Pan, Q. Xu, Y. Lu, H. Xu, Y. Sun, N. Dai, and J. Hao, "Wide gamut, angle-insensitive structural colors based on deepsubwavelength bilayer media," Nanophotonics 9, 3385 (2020).

2. W. Yang, S. Xiao, Q. Song, Y. Liu, Y. Wu, S. Wang, J. Yu, J. Han, and D. P. Tsai, "All-dielectric metasurface for high-performance structural color," Nat. Commun. 11, 1864 (2020).

3. M. Song, D. Wang, S. Peana, S. Choudhury, P. Nyga, Z. A. Kudyshev, H. Yu, A. Boltasseva, V. M. Shalaev, and A. V. Kildishev, "Colors with plasmonic nanostructures: a full-spectrum review,” Appl. Phys. Rev. 6, 041308 (2019).

4. H. Galinski, G. Favraud, H. Dong, J. S. T. Gongora, G. Favaro, M. Dobeli, R. Spolenak, A. Fratalocchi, and F. Capasso, "Scalable, ultra-resistant structural colors based on network metamaterials," Light Sci. Appl. 6, e16233 (2017). 
5. Y. Li, J. Hu, W. Liu, J. Yin, and J. Lu, "High period frequency LIPSS emerging on 304 stainless steel under the irradiation of femtosecond laser double-pulse trains," Chin. Opt. Lett. 19, 123801 (2021).

6. T. Jiang, S. Gao, Z. Tian, H. Zhang, and L. Niu, "Fabrication of diamond ultrafine structures by femtosecond laser," Chin. Opt. Lett. 18, 101402 (2020).

7. B. Wu, C. Wang, Z. Luo, J. Li, S. Man, K. Ding, and J. A. Duan, "Controllable annulus micro-/nanostructures on copper fabricated by femtosecond laser with spatial doughnut distribution," Chin. Opt. Lett. 18, 013101 (2020).

8. J. Yao, C. Zhang, H. Liu, Q. Dai, L. Wu, S. Lan, A. V. Gopal, V. A. Trofimov, and T. M. Lysak, "Selective appearance of several laser-induced periodic surface structure patterns on a metal surface using structural colors produced by femtosecond laser pulses," Appl. Surf. Sci. 258, 7625 (2012).

9. Z. Ou, M. Huang, and F. Zhao, "Colorizing pure copper surface by ultrafast laser-induced near-subwavelength ripples," Opt. Express 22, 17254 (2014).

10. K. Cao, L. Chen, H. Wu, J. Liu, K. Cheng, Y. Li, Y. Xia, C. Feng, S. Zhang, D. Feng, Z. Sun, and T. Jia, "Large-area commercial-grating-quality subwavelength periodic ripples on silicon efficiently fabricated by gentle ablation with femtosecond laser interference via two cylindrical lenses," Opt. Laser. Technol. 131, 106441 (2020).

11. F. Luo, W. Ong, Y. Guan, F. Li, S. Sun, G. C. Lim, and M. Hong, "Study of micro/nanostructures formed by a nanosecond laser in gaseous environments for stainless steel surface coloring," Appl. Surf. Sci. 328, 405 (2015).

12. B. Zhang, D. Tan, X. Liu, L. Tong, P. G. Kazansky, and J. Qiu, "Self-organized periodic crystallization in unconventional glass created by an ultrafast laser for optical attenuation in the broadband near-infrared region," Adv. Opt. Mater. 7, 1900593 (2019).

13. M. Wang and Y. Yin, "Magnetically responsive nanostructures with tunable optical properties," J. Am. Chem. Soc. 138, 6315 (2016).

14. X. Wang, Z. Wang, L. Bai, H. Wang, L. Kang, D. Werner, M. Xu, B. Li, J. Li, and $\mathrm{X}$. Yu, "Vivid structural colors from long-range ordered and carbonintegrated colloidal photonic crystals," Opt. Express 26, 27001 (2018).

15. D. Tan, B. Zhang, and J. Qiu, "Ultrafast laser direct writing in glass: thermal accumulation engineering and applications," Laser Photonics Rev. 15, 2000455 (2021).

16. B. Öktem, I. Pavlov, S. Ilday, H. Kalaycıoğlu, A. Rybak, S. Yavaş, M. Erdoğan, and F. Ö. Ilday, "Nonlinear laser lithography for indefinitely large-area nanostructuring with femtosecond pulses," Nat. Photonics 7, 897 (2013).

17. J. Gottmann and R. Wagner, "Sub-wavelength ripple formation on various materials induced by tightly focused femtosecond laser radiation," Proc. SPIE 6106, 61061R (2006)
18. X. Zhu, I. Czolkos, A. Johansson, T. Nielsen, and A. Kristensen, "Master origination by $248 \mathrm{~nm}$ DUV lithography for plasmonic color generation," Appl. Phys. Lett. 118, 141103 (2021).

19. F. Shu, F. Yu, R. Peng, Y. Zhu, B. Xiong, R. Fan, Z. Wang, Y. Liu, and M. Wang, "Dynamic plasmonic color generation based on phase transition of vanadium dioxide," Adv. Opt. Mater. 6, 1700939 (2018).

20. D. Hu, Y. Lu, Y. Cao, Y. Zhang, Y. Xu, W. Li, F. Gao, B. Cai, B. O. Guan, C. W. Qiu, and X. Li, "Laser-splashed three-dimensional plasmonic nanovolcanoes for steganography in angular anisotropy," ACS Nano 12, 9233 (2018).

21. Z. Yang, C. Ji, D. Liu, and L. J. Guo, "Enhancing the purity of reflective structural colors with ultrathin bilayer media as effective ideal absorbers," Adv. Opt. Mater. 7, 1900739 (2019).

22. J. Liu, K. Feng, Y. Wang, Q. Li, N. Chen, and Y. Bu, "High-color-purity, highbrightness and angle-insensitive red structural color," Chin. Opt. Lett. 20, 021601 (2022).

23. J. Chu, J. Wang, J. Wang, X. Liu, Y. Zhang, L. Shi, and J. Zi, "Structuralcolored silk based on Ti-Si bilayer," Chin. Opt. Lett. 19, 051601 (2021).

24. J. Wang, Q. Guo, X. Liu, Y. Dai, Z. Wang, and J. Qiu, "Bubble generation in germanate glass induced by femtosecond laser," Chin. Phys. Lett. 33, 036101 (2016).

25. J. Chelikowsk, D. Chad, and N. Binggeli, "Ultrafast laser-induced modification of optical glasses: a spectroscopy insight into the microscopic mechanisms," Phys. Rev. B 62, R2251 (2000).

26. J. Zhang, M. Gecevicius, M. Beresna, and P. G. Kazansky, "Seemingly unlimited lifetime data storage in nanostructured glass," Phys. Rev. Lett. 112, 033901 (2014)

27. D. Tan, X. Sun, Q. Wang, P. Zhou, Y. Liao, and J. Qiu, "Fabricating low loss waveguides over a large depth in glass by temperature gradient assisted femtosecond laser writing," Opt. Lett. 45, 3941 (2020).

28. D. Tan, Z. Wang, B. Xu, and J. Qiu, "Photonic circuits written by femtosecond laser in glass: improved fabrication and recent progress in photonic devices," Adv. Photonics 3, 024002 (2021).

29. J. Wu, Y. Zhang, L. Li, Y. Ren, Q. Lu, L. Wang, and F. Chen, "Raman spectra study on modifications of BK7 glass induced by 1030-nm and 515-nm femtosecond laser," Results Phys. 21, 103814 (2021).

30. K. Yoon, S. Choi, J. Paek, D. Im, J. Roh, J. Kwon, and H. Kim, "Iridescent specular structural colors of two-dimensional periodic diffraction gratings," J. Opt. Soc. Korea 18, 616 (2014). 